\title{
Dietary and nutritional approaches for prevention and management of type 2 diabetes
}

\author{
(c) (1) @ $\circledast$ OPEN ACCESS
}

Common ground on dietary approaches for the prevention, management, and potential remission of type 2 diabetes can be found, argue Nita G Forouhi and colleagues

\author{
Nita G Forouhi professor ${ }^{1}$, Anoop Misra professor ${ }^{2}$, Viswanathan Mohan professor ${ }^{3}$, Roy Taylor \\ professor $^{4}$, William Yancy director ${ }^{5} 6$
}

${ }^{1}$ MRC Epidemiology Unit, University of Cambridge School of Clinical Medicine, Cambridge, UK; ${ }^{2}$ Fortis-C-DOC Centre of Excellence for Diabetes, Metabolic Diseases and Endocrinology, and National Diabetes, Obesity and Cholesterol Foundation, New Delhi, India; ${ }^{3}$ Dr Mohan's Diabetes Specialities Centre and Madras Diabetes Research Foundation, Chennai, India; ${ }^{4}$ Magnetic Resonance Centre, Institute of Cellular Medicine, Newcastle University, Newcastle, UK; ${ }^{5}$ Duke University Diet and Fitness Center, Durham, North Carolina, USA; ${ }^{6}$ Department of Medicine, Duke University School of Medicine, Durham, North Carolina, USA; ${ }^{7}$ Center for Health Services Research in Primary Care, Department of Veterans Affairs, Durham, North Carolina, USA

Dietary factors are of paramount importance in the management and prevention of type 2 diabetes. Despite progress in formulating evidence based dietary guidance, controversy and confusion remain. In this article, we examine the evidence for areas of consensus as well as ongoing uncertainty or controversy about dietary guidelines for type 2 diabetes. What is the best dietary approach? Is it possible to achieve remission of type 2 diabetes with lifestyle behaviour changes or is it inevitably a condition causing progressive health decline? We also examine the influence of nutrition transition and population specific factors in the global context and discuss future directions for effective dietary and nutritional approaches to manage type 2 diabetes and their implementation.

\section{Why dietary management matters but is difficult to implement}

Diabetes is one of the biggest global public health problems: the prevalence is estimated to increase from 425 million people in 2017 to 629 million by 2045, with linked health, social, and economic costs. ${ }^{1}$ Urgent solutions for slowing, or even reversing, this trend are needed, especially from investment in modifiable factors including diet, physical activity, and weight. Diet is a leading contributor to morbidity and mortality worldwide according to the Global Burden of Disease Study carried out in 188 countries. $^{2}$ The importance of nutrition in the management and prevention of type 2 diabetes through its effect on weight and metabolic control is clear. However, nutrition is also one of the most controversial and difficult aspects of the management of type 2 diabetes.

The idea of being on a "diet" for a chronic lifelong condition like diabetes is enough to put many people off as knowing what to eat and maintaining an optimal eating pattern are challenging. Medical nutrition therapy was introduced to guide a systematic and evidence based approach to the management of diabetes through diet, and its effectiveness has been demonstrated, ${ }^{3}$ but difficulties remain. Although most diabetes guidelines recommend starting pharmacotherapy only after first making nutritional and physical activity lifestyle changes, this is not always followed in practice globally. Most physicians are not trained in nutrition interventions and this is a barrier to counselling patients. ${ }^{45}$ Moreover, talking to patients about nutrition is time consuming. In many settings, outside of specialised diabetes centres where trained nutritionists/educators are available, advice on nutrition for diabetes is, at best, a printed menu given to the patient. In resource poor settings, when type 2 diabetes is diagnosed, often the patient leaves the clinic with a list of new medications and little else. There is wide variation in the use of dietary modification alone to manage type 2 diabetes: for instance, estimates of fewer than 5-10\% of patients with type 2 diabetes in India ${ }^{6}$ and $31 \%$ in the UK are reported, although patients treated by lifestyle measures may be less closely managed than patients on medication for type 2 diabetes. ${ }^{7}$ Although systems are usually in place to record and monitor process measures for diabetes care in medical records, dietary 
information is often neglected, even though at least modest attention to diet is needed to achieve adequate glycaemic control. Family doctors and hospital clinics should collect this information routinely but how to do this is a challenge. ${ }^{58}$

Progress has been made in understanding the best dietary advice for diabetes but broader problems exist. For instance, increasing vegetable and fruit intake is recommended by most dietary guidelines but their cost is prohibitively high in many settings: the cost of two servings of fruits and three servings of vegetables a day per individual (to fulfil the " 5 -a-day" guidance) accounted for $52 \%, 18 \%, 16 \%$, and $2 \%$ of household income in low, low to middle, upper to middle, and high income countries, respectively. ${ }^{9}$ An expensive market of foods labelled for use by people with diabetes also exists, with products often being no healthier, and sometimes less healthy, than regular foods. After new European Union legislation, food regulations in some countries, including the UK, were updated as recently as July 2016 to ban such misleading labels. This is not the case elsewhere, however, and what will happen to such regulation after the UK leaves the European Union is unclear, which highlights the importance of the political environment.

\section{Evidence for current dietary guidelines}

In some, mostly developed, countries, dietary guidelines for the management of diabetes have evolved from a focus on a low fat diet to the recognition that more important considerations are macronutrient quality (that is, the type versus the quantity of macronutrient), avoidance of processed foods (particularly processed starches and sugars), and overall dietary patterns. Many systematic reviews and national dietary guidelines have evaluated the evidence for optimal dietary advice, and we will not repeat the evidence review. ${ }^{10-18} \mathrm{We}$ focus instead in the following sections on some important principles where broad consensus exists in the scientific and clinical community and highlight areas of uncertainty, but we begin by outlining three underpinning features.

Firstly, an understanding of healthy eating for the prevention and management of type 2 diabetes has largely been derived from long term prospective studies and limited evidence from randomised controlled trials in general populations,

supplemented by evidence from people with type 2 diabetes. Many published guidelines and reviews have applied grading criteria and this evidence is often of moderate quality in the hierarchy of evidence that places randomised controlled trials at the top. Elsewhere, it is argued that different forms of evidence evaluating consistency across multiple study designs including large population based prospective studies of clinical endpoints, controlled trials of intermediate pathways, and where feasible randomised trials of clinical endpoints should be used collectively for evidence based nutritional guidance. ${ }^{19}$

Secondly, it is now recognised that dietary advice for both the prevention and management of type 2 diabetes should converge, and they should not be treated as different entities (fig 1).

However, in those with type 2 diabetes, the degree of glycaemic control and type and dose of diabetes medication should be coordinated with dietary intake. ${ }^{12}$ With some dietary interventions, such as very low calorie or low carbohydrate diets, people with diabetes would usually stop or reduce their diabetes medication and be monitored closely, as reviewed in a later section.

Thirdly, while recognising the importance of diet for weight management, there is now greater understanding ${ }^{10}$ of the multiple pathways through which dietary factors exert health effects through both obesity dependent and obesity independent mechanisms. The influence of diet on weight, glycaemia, and glucose-insulin homeostasis is directly relevant to glycaemic control in diabetes, while other outcomes such as cardiovascular complications are further influenced by the effect of diet on blood lipids, apolipoproteins, blood pressure, endothelial function, thrombosis, coagulation, systemic inflammation, and vascular adhesion. The effect of food and nutrients on the gut microbiome may also be relevant to the pathogenesis of diabetes but further research is needed. Therefore, diet quality and quantity over the longer term are relevant to the prevention and management of diabetes and its complications through a wide range of metabolic and physiological processes.

\section{Areas of consensus in guidelines Weight management}

Type 2 diabetes is most commonly associated with overweight or obesity and insulin resistance. Therefore, reducing weight and maintaining a healthy weight is a core part of clinical management. Weight loss is also linked to improvements in glycaemia, blood pressure, and lipids and hence can delay or prevent complications, particularly cardiovascular events.

\section{Energy balance}

Most guidelines recommend promoting weight loss among overweight or obese individuals by reducing energy intake. Portion control is one strategy to limit energy intake together with a healthy eating pattern that focuses on a diet composed of whole or unprocessed foods combined with physical activity and ongoing support.

\section{Dietary patterns}

The evidence points to promoting patterns of food intake that are high in vegetables, fruit, whole grains, legumes, nuts, and dairy products such as yoghurt but with some cautions. Firstly, some dietary approaches (eg, low carbohydrate diets) recommend restricting the intake of fruits, whole grains, and legumes because of their sugar or starch content. For fruit intake, particularly among those with diabetes, opinion is divided among scientists and clinicians (see appendix on bmj.com). Many guidelines continue to recommend fruit, however, on the basis that fructose intake from fruits is preferable to isocaloric intake of sucrose or starch because of the additional micronutrient, phytochemical, and fibre content of fruit. Secondly, despite evidence from randomised controlled trials and prospective studies ${ }^{10}$ that nuts may help prevent type 2 diabetes, some (potentially misplaced) concern exists about their high energy content. Further research in people with type 2 diabetes should help to clarify this.

There is also consensus on the benefits of certain named dietary patterns such as the Mediterranean diet for prevention and management of type 2 diabetes. Expert guidelines also support other healthy eating patterns that take account of local sociocultural factors and personal preferences.

\section{Foods to avoid}

Consensus exists on reducing or avoiding the intake of processed red meats, refined grains and sugars (especially sugar sweetened drinks) both for prevention and management of type 2 diabetes, again with some cautions. Firstly, for unprocessed red meat, the evidence of possible harm because of the development of type 2 diabetes is less consistent and of a smaller magnitude. More research is needed on specific benefits or harms in people with type 2 diabetes. Secondly, evidence is increasing on the 
relevance of carbohydrate quality: that is that whole grains and fibre are better choices than refined grains and that fibre intake should be at least as high in people with type 2 diabetes as recommended for the general population, that diets that have a higher glycaemic index and load are associated with an increased risk of type 2 diabetes, and that there is a modest glycaemic benefit in replacing foods with higher glycaemic load with foods with low glycaemic load. However, debate continues about the independence of these effects from the intake of dietary fibre. Some evidence exists that consumption of potato and white rice may increase the risk of type 2 diabetes but this is limited and further research is needed.

Moreover, many guidelines also highlight the importance of reducing the intake of in foods high in sodium and trans fat because of the relevance of these specifically for cardiovascular health.

\section{Areas of uncertainty in guidelines Optimal macronutrient composition}

One of the most contentious issues about the management of type 2 diabetes has been on the best macronutrient composition of the diet. Some guidelines continue to advise macronutrient quantity goals, such as the European or Canadian recommendation of 45-60\% of total energy as carbohydrate, $10-20 \%$ as protein, and less than $35 \%$ as fat, ${ }^{1320}$ or the Indian guidelines that recommend 50-60\% energy from carbohydrates, $10-15 \%$ from protein, and less than $30 \%$ from fat. ${ }^{21}$ In contrast, the most recent nutritional guideline from the American Diabetes Association concluded that there is no ideal mix of macronutrients for all people with diabetes and recommended individually tailored goals. ${ }^{12}$ Alternatively, a low carbohydrate diet for weight and glycaemic control has gained popularity among some experts, clinicians, and the public (reviewed in a later section). Others conclude that a low carbohydrate diet combined with low saturated fat intake is best. ${ }^{22}$

For weight loss, three points are noteworthy when comparing dietary macronutrient composition. Firstly, evidence from trials points to potentially greater benefits from a low carbohydrate than a low fat diet but the difference in weight loss between diets is modest. ${ }^{23}$ Secondly, a comparison of named diet programmes with different macronutrient composition highlighted that the critical factor in effectiveness for weight loss was the level of adherence to the diet over time. ${ }^{24}$ Thirdly, the quality of the diet in low carbohydrate or low fat diets is important. $^{25} 26$

Research to date on weight or metabolic outcomes in diabetes is complicated by the use of different definitions for the different macronutrient approaches. For instance, the definition of a low carbohydrate diet has ranged from $4 \%$ of daily energy intake from carbohydrates (promoting nutritional ketosis) to $40 \% .{ }^{15}$ Similarly, low fat diets have been defined as fat intake less than $30 \%$ of daily energy intake or substantially lower. Given these limitations, the best current approach may be an emphasis on the use of individual assessment for dietary advice and a focus on the pattern of eating that most readily allows the individual to limit calorie intake and improve macronutrient quality (such as avoiding refined carbohydrates).

\section{Fish}

Regular fish intake of at least two servings a week, including one serving of oily fish (eg, salmon, mackerel, and trout) is recommended for cardiovascular risk prevention but fish intake has different associations with the risk of developing type 2 diabetes across the world-an inverse association, no association, and a positive association. ${ }^{27}$ It is thought that the type of fish consumed, preparation or cooking practices, and possible contaminants (eg, methyl mercury and polychlorinated biphenyls) vary by geographical location and contributed to this heterogeneity. More research is needed to resolve whether fish intake should be recommended for the prevention of diabetes. However, the current evidence supports an increase in consumption of oily fish for individuals with diabetes because of its beneficial effects on lipoproteins and prevention of coronary heart disease. Most guidelines agree that omega 3 polyunsaturated fatty acid (fish oil) supplementation for cardiovascular prevention in people with diabetes should not be recommended but more research is needed and the results of the ASCEND (A Study of Cardiovascular Events in Diabetes) trial should help to clarify this. ${ }^{28}$

\section{Dairy}

Dairy foods are encouraged for the prevention of type 2 diabetes, with more consistent evidence of the benefits of fermented dairy products, such as yoghurt. Similar to population level recommendations about limiting the intake of foods high in saturated fats and replacing them with foods rich in polyunsaturated fat, the current advice for diabetes also favours low fat dairy products but this is debated. More research is needed to resolve this question.

\section{Oils}

Uncertainty continues about certain plant oils and tropical oils such as coconut or palm oil as evidence from prospective studies or randomised controlled trials on clinical events is sparse or non-existent. However, olive oil, particularly extra virgin olive oil, has been studied in greater detail with evidence of potential benefits for the prevention and management of type 2 diabetes ${ }^{29}$ and the prevention of cardiovascular disease within the context of a Mediterranean $\operatorname{diet}^{30}$ (see article in this series on dietary fats). ${ }^{31}$

\section{Difficulties in setting guidelines}

Where dietary guidelines exist (in many settings there are none, or they are adapted from those in developed countries and therefore may not be applicable to the local situation), they vary substantially in whether they are evidence based or opinion pieces, and updated in line with scientific progress or outdated. Their accessibility—both physical availability (eg, through a website or clinic) and comprehensibility - for patients and healthcare professionals varies. They vary also in scope, content, detail, and emphasis on the importance of individualised dietary advice, areas of controversy, and further research needs. The quality of research that informs dietary guidelines also needs greater investment from the scientific community and funders. Moreover, lack of transparency in the development of guidelines and bias in the primary nutritional studies can undermine the development of reliable dietary guidelines; recommendations for their improvement must be heeded. ${ }^{32}$

\section{Reversing type 2 diabetes through diet}

Type 2 diabetes was once thought to be irreversible and progressive after diagnosis, but much interest has arisen about the potential for remission. Consensus on the definition of remission is a sign of progress: glucose levels lower than the diagnostic level for diabetes in the absence of medications for hyperglycaemia for a period of time (often proposed to be at 
least one year). ${ }^{334}$ However, the predominant role of energy deficit versus macronutrient composition of the diet in achieving remission is still controversial.

\section{Remission through a low calorie energy deficit diet}

Although the clinical observation of the lifelong, steadily progressive nature of type 2 diabetes was confirmed by the UK Prospective Diabetes Study, ${ }^{35}$ rapid normalisation of fasting plasma glucose after bariatric surgery suggested that deterioration was not inevitable. ${ }^{36}$ As the main change was one of sudden calorie restriction, a low calorie diet was used as a tool to study the mechanisms involved. In one study of patients with type 2 diabetes, fasting plasma glucose normalised within seven days of following a low calorie diet. ${ }^{37}$ This normalisation through diet occurred despite simultaneous withdrawal of metformin therapy. Gradually over eight weeks, glucose stimulated insulin secretion returned to normal. ${ }^{37}$ Was this a consequence of calorie restriction or composition of the diet? To achieve the degree of weight loss obtained $(15 \mathrm{~kg})$, about $610 \mathrm{kcal}$ a day was provided- $510 \mathrm{kcal}$ as a liquid formula diet and about $100 \mathrm{kcal}$ as non-starchy vegetables. The formula diet consisted of $59 \mathrm{~g}$ of carbohydrate ( $30 \mathrm{~g}$ as sugars), $11.4 \mathrm{~g}$ of fat, and $41 \mathrm{~g}$ of protein, including required vitamins and minerals. This high "sugar" approach to controlling blood glucose may be surprising but the critical aspect is not what is eaten but the gap between energy required and taken in. Because of this deficit, the body must use previously stored energy. Intrahepatic fat is used first, and the $30 \%$ decrease in hepatic fat in the first seven days appears sufficient to normalise the insulin sensitivity of the liver. ${ }^{37}$ In addition, pancreatic fat content fell over eight weeks and beta cell function improved. This is because insulin secretory function was regained by re-differentiation after fat removal. $^{38}$

The permanence of these changes was tested by a nutritional and behavioural approach to achieve long term isocaloric eating after the acute weight loss phase. ${ }^{39}$ It was successful in keeping weight steady over the next six months of the study. Calorie restriction was associated with both hepatic and pancreatic fat content remaining at the low levels achieved. The initial remission of type 2 diabetes was closely associated with duration of diabetes, and the individuals with type 2 diabetes of shorter duration who achieved normal levels of blood glucose maintained normal physiology during the six month follow-up period. Recently, $46 \%$ of a UK primary care cohort remained free of diabetes at one year during a structured low calorie weight loss programme (the DiRECT trial). ${ }^{40}$ These results are convincing, and four years of follow-up are planned.

A common criticism of the energy deficit research has been that very low calorie diets may not be achievable or sustainable. Indeed, adherence to most diets in the longer term is an important challenge. ${ }^{24}$ However, Look-AHEAD, the largest randomised study of lifestyle interventions in type 2 diabetes $(\mathrm{n}=5145)$, randomised individuals to intensive lifestyle management, including the goal to reduce total calorie intake to $1200-1800 \mathrm{kcal} / \mathrm{d}$ through a low fat diet assisted by liquid meal replacements, and this approach achieved greater weight loss and non-diabetic blood glucose levels at year 1 and year 4 in the intervention than the control group. ${ }^{41}$

Considerable interest has arisen about whether low calorie diets associated with diabetes remission can also help to prevent diabetic complications. Evidence is sparse because of the lack of long term follow-up studies but the existing research is promising. A return to the non-diabetic state brings an improvement in cardiovascular risk ( $\mathrm{Q}$ risk decreasing from $19.8 \%$ to $5.4 \%)^{39}$; case reports of individuals facing foot amputation record a return to a low risk state over 2-4 years with resolution of painful neuropathy ${ }^{4243}$; and retinal complications are unlikely to occur or progress. ${ }^{44}$ However, other evidence highlights that worsening of treatable maculopathy or proliferative retinopathy may occur following a sudden fall in plasma glucose levels, ${ }^{456}$ so retinal imaging in 4-6 months is recommended for individuals with more than minimal retinopathy if following a low calorie remission diet. Annual review is recommended for all those in the post-diabetic state, and a "diabetes in remission" code (C10P) is now available in the $\mathrm{UK}^{34}$

\section{Management or remission through a low carbohydrate diet}

Before insulin was developed as a therapy, reducing carbohydrate intake was the main treatment for diabetes. ${ }^{47} 48$ Carbohydrate restriction for the treatment of type 2 diabetes has been an area of intense interest because, of all the macronutrients, carbohydrates have the greatest effect on blood glucose and insulin levels. ${ }^{49}$

In a review by the American Diabetes Association, interventions of low carbohydrate (less than $40 \%$ of calories) diets published from 2001 to 2010 were identified..$^{15}$ Of 11 trials, eight were randomised and about half reported greater improvement in $\mathrm{HbA1c}$ on the low carbohydrate diet than the comparison diet (usually a low fat diet), and a greater reduction in the use of medicines to lower glucose. Notably, calorie reduction coincided with carbohydrate restriction in many of the studies, even though it was not often specified in the dietary counselling. One of the more highly controlled studies was an inpatient feeding study, ${ }^{50}$ which reported a decline in mean $\mathrm{HbA} 1 \mathrm{c}$ from $7.3 \%$ to $6.8 \%$ $(\mathrm{P}=0.006)$ over just 14 days on a low carbohydrate diet.

For glycaemia, other reviews of evidence from randomised trials on people with type 2 diabetes have varying conclusions. ${ }^{51-56}$ Some concluded that low carbohydrate diets were superior to other diets for glycaemic control, or that a dose response relationship existed, with stricter low carbohydrate restriction resulting in greater reductions in glycaemia. Others cautioned about short term beneficial effects not being sustained in the longer term, or found no overall advantage over the comparison diet. Narrative reviews have generally been more emphatic on the benefits of low carbohydrate diets, including increased satiety, and highlight the advantages for weight loss and metabolic parameters. ${ }^{57} 58$ More recently, a one year clinic based study of the low carbohydrate diet designed to induce nutritional ketosis (usually with carbohydrate intake less than $30 \mathrm{~g} / \mathrm{d}$ ) was effective for weight loss, and for glycaemic control and medication reduction. ${ }^{59}$ However, the study was not randomised, treatment intensity differed substantially in the intervention versus usual care groups, and participants were able to select their group.

Concerns about potential detrimental effects on cardiovascular health have been raised as low carbohydrate diets are usually high in dietary fat, including saturated fat. For lipid markers as predictors of future cardiovascular events, several studies found greater improvements in high density lipoprotein cholesterol and triglycerides with no relative worsening of low density lipoprotein cholesterol in patients with type 2 diabetes following carbohydrate restriction, ${ }^{15}$ with similar conclusions in non-diabetic populations. ${ }^{57}$ 60-62 Low density lipoprotein cholesterol tends to decline more, however, in a low fat comparison $\operatorname{diet}^{6163}$ and although low density lipoprotein 
cholesterol may not worsen with a low carbohydrate $\operatorname{diet}^{63}$ in the short term, the longer term effects are unclear. Evidence shows that low carbohydrate intake can lower the more atherogenic small, dense low density lipoprotein particles. ${ }^{5764}$ Because some individuals may experience an increase in serum low density lipoprotein cholesterol when following a low carbohydrate diet high in saturated fat, monitoring is important.

Another concern is the effect of the potentially higher protein content of low carbohydrate diets on renal function. Evidence from patients with type 2 diabetes with normal baseline renal function and from individuals without diabetes and with normal or mildly impaired renal function has not shown worsening renal function at one or up to two years of follow-up, respectively. ${ }^{225-67}$ Research in patients with more severely impaired renal function, with or without diabetes, has not been reported to our knowledge. Other potential side effects of a very low carbohydrate diet include headache, fatigue, and muscle cramping but these side effects can be avoided by adequate fluid and sodium intake, particularly in the first week or two after starting the diet when diuresis is greatest. Concern about urinary calcium loss and a possible contribution to increased future risk of kidney stones or osteoporosis ${ }^{68}$ have not been verified ${ }^{69}$ but evidence is sparse and warrants further investigation. The long term effects on cardiovascular disease and chronic kidney disease in patients with diabetes need further evaluation.

Given the hypoglycaemic effect of carbohydrate restriction, patients with diabetes who adopt low carbohydrate diets and their clinicians must understand how to avoid hypoglycaemia by appropriately reducing glucose lowering medications. Finally, low carbohydrate diets can restrict whole grain intake and although some low carbohydrate foods can provide the fibre and micronutrients contained in grains, it may require greater effort to incorporate such foods. This has led some experts to emphasise restricting refined starches and sugars but retaining whole grains.

\section{Nutrition transition and population specific factors}

Several countries in sub-Saharan Africa, South America, and Asia (eg, India and China) have undergone rapid nutrition transition in the past two decades. These changes have paralleled economic growth, foreign investment in the fast food industry, urbanisation, direct-to-consumer marketing of foods high in calories, sale of ultraprocessed foods, and as a result, lower consumption of traditional diets. The effect of these factors on nutrition have led to obesity and type 2 diabetes on the one hand, and co-existing undernutrition and micronutrient deficiencies on the other.

Dietary shifts in low and middle income countries have been stark: in India, these include a substantial increase in fat intake in the setting of an already high carbohydrate intake, with a slight increase in total energy and protein, ${ }^{70}$ and a decreasing intake of coarse cereals, pulses, fruits, and vegetables ${ }^{71}$; in China, animal protein and fat as a percentage of energy has also increased, while cereal intake has decreased. ${ }^{72}$ An almost universal increase in the intake of caloric beverages has also occurred, with sugar sweetened soda drinks being the main beverage contributing to energy intake, for example among adults and children in Mexico, ${ }^{73}$ or the substantial rise in China in sales of sugar sweetened drinks from $10.2 \mathrm{~L}$ per capita in 1998 to 55.0 L per capita in $2012 .^{74}$ The movement of populations from rural to urban areas within a country may also be linked with shifts in diets to more unhealthy patterns, ${ }^{75}$ while acculturation of immigrant populations into their host countries also results in dietary shifts. ${ }^{76}$

In some populations, such as South Asians, rice and wheat flour bread are staple foods, with a related high carbohydrate intake (60-70\% of calories). ${ }^{77}$ Although time trends show that intake of carbohydrate has decreased among South Asian Indians, the quality of carbohydrates has shifted towards use of refined carbohydrates. ${ }^{71}$ The use of oils and traditional cooking practices also have specific patterns in different populations. For instance, in India, the import and consumption of palm oil, often incorporated in the popular oil vanaspati (partially hydrogenated vegetable oil, high in trans fats), is high. ${ }^{78}$ Moreover, the traditional Indian cooking practice of frying at high temperatures and re-heating increases trans fatty acids in oils. ${ }^{79}$ Such oils are low cost, readily available, and have a long shelf life, and thus are more attractive to people from the middle and low socioeconomic strata but their long term effects on type 2 diabetes are unknown.

Despite the nutrition transition being linked to an increasing prevalence of type 2 diabetes, obesity and other non-communicable diseases, strong measures to limit harmful foods are not in place in many countries. Regulatory frameworks including fiscal policies such as taxation for sugar sweetened beverages need to be strengthened to be effective and other preventive interventions need to be properly implemented. Efforts to control trans fatty acids in foods have gained momentum but are largely confined to developed countries. To reduce consumption in low and middle income countries will require both stringent regulations and the availability and development of alternative choices of healthy and low cost oils, ready made food products, and consumer education. ${ }^{80}$ The need for nutritional labelling is important but understanding nutrition labels is a problem in populations with low literacy or nutrition awareness, which highlights the need for educational activities and simpler forms of labelling. The role of dietary/nutritional factors in the predisposition of some ethnic groups to developing type 2 diabetes at substantially lower levels of obesity than European populations ${ }^{81}$ is poorly researched and needs investigation.

\section{Conclusion}

Despite the challenges of nutritional research, considerable progress has been made in formulating evidence based dietary guidance and some common principles can be agreed that should be helpful to clinicians, patients, and the public. Several areas of uncertainty and controversy remain and further research is needed to resolve these. While adherence to dietary advice is an important challenge, weight management is still a cornerstone in diabetes management, supplemented with new developments, including the potential for the remission of type 2 diabetes through diet. 


\section{Future directions}

Nutritional research is difficult. Although much progress has been made to improve evidence based dietary guidelines, more investment is needed in good quality research with a greater focus on overcoming the limitations of existing research. Experts should also strive to build consensus using research evidence based on a combination of different study designs, including randomised experiments and prospective observational studies

- High quality research is needed that compares calorie restriction and carbohydrate restriction to assess effectiveness and feasibility in the long term. Consensus is needed on definitions of low carbohydrate nutrition. Use of the findings must take account of individual preferences, whole diets, and eating patterns

- Further research is needed to resolve areas of uncertainty about dietary advice in diabetes, including the role of nuts, fruits, legumes, fish, plan oils, low fat versus high fat dairy, and diet quantity and quality

- Given recent widespread recommendations (such as from the World Health Organization ${ }^{82}$ and the UK Scientific Advisory Committee on Nutrition $^{83}$ ) to reduce free sugars to under $10 \%$ or even $5 \%$ of total energy intake in the general population and to avoid sugar sweetened drinks, we need targeted research on the effect of non-nutritive sweeteners on health outcomes in people with diabetes and in the whole population

- Most dietary guidelines are derived from evidence from Western countries. Research is needed to better understand the specific aetiological factors that link diet/nutrition and diabetes and its complications in different regions and different ethnic groups. This requires investment in developing prospective cohorts and building capacity to undertake research in low and middle income settings and in immigrant ethnic groups. Up-to-date, evidence based dietary guidelines are needed that are locally relevant and readily accessible to healthcare professionals, patients, and the public in different regions of the world. Greater understanding is also needed about the dietary determinants of type 2 diabetes and its complications at younger ages and in those with lower body mass index in some ethnic groups

- We need investment in medical education to train medical students and physicians in lifestyle interventions, including incorporating nutrition education in medical curricula

- Individual, collective, and upstream factors are important. Issuing dietary guidance does not ensure its adoption or implementation. Research is needed to understand the individual and societal drivers of and barriers to healthy eating. Educating and empowering individuals to make better dietary choices is an important strategy; in particular, the social aspects of eating need attention as most people eat in family or social groups and counselling needs to take this into account. Equally important is tackling the wider determinants of individual behaviour-the "foodscape", sociocultural and political factors, globalisation, and nutrition transition

\section{Key messages}

Considerable evidence supports a common set of dietary approaches for the prevention and management of type 2 diabetes, but uncertainties remain
reme

- Weight management is a cornerstone of metabolic health but diet quality is also important

- Low carbohydrate diets as the preferred choice in type 2 diabetes is controversial. Some guidelines maintain that no single ideal percentage distribution of calories from different macronutrients (carbohydrates, fat, or protein) exists, but there are calls to review this in light of emerging evidence on the potential benefits of low carbohydrate diets for weight management and glycaemic control

- The quality of carbohydrates such as refined versus whole grain sources is important and should not get lost in the debate on quantity

- Recognition is increasing that the focus of dietary advice should be on foods and healthy eating patterns rather than on nutrients. Evidence supports avoiding processed foods, refined grains, processed red meats, and sugar sweetened drinks and promoting the intake of fibre, vegetables, and yoghurt. Dietary advice should be individually tailored and take into account personal, cultural, and social factors

- An exciting recent development is the understanding that type 2 diabetes does not have to be a progressive condition but instead there is potential for remission with dietary intervention

We thank Sue Brown as a patient representative of Diabetes UK for her helpful comments and insight into this article.

Contributors and sources: The authors have experience and research interests in the prevention and management of type 2 diabetes (NGF, AM, VM, RT, WY), in guideline development (NGF, AM, VM, WY), and in nutritional epidemiology (NGF, VM). Sources of information for this article included published dietary guidelines or medical nutrition therapy guidelines for diabetes, and systematic reviews and primary research articles based on randomised clinical trials or prospective observational studies. All authors contributed to drafting this manuscript, with NGF taking a lead role and she is also the guarantor of the manuscript. All authors gave intellectual input to improve the manuscript and have read and approved the final version.

Competing interests: We have read and understood BMJ policy on declaration of interests and declare the following: NGF receives funding from the Medical Research Council Epidemiology Unit (MC_UU_12015/5). NGF is a member (unpaid) of the Joint SACN/NHS-England/Diabetes-UK Working Group to review the evidence on lower carbohydrate diets compared with current government advice for adults with type 2 diabetes and is a member (unpaid) of ILSI-Europe Qualitative Fat Intake Task Force Expert Group on update on health effects of different saturated fats. AM received honorarium and research funding from Herbalife and Almond Board of California. VM has received funding from Abbott Health Care for meal replacement studies, the Cashew Export Promotion Council of India, and the Almond Board of California for studies on nuts. RT has received funding from Diabetes UK for the Diabetes Remission Clinical Trial and he is a member (unpaid) of the Joint SACN/NHS-England/Diabetes-UK Working Group to review the evidence on lower carbohydrate diets compared to current government advice for adults with type 2 diabetes. WY has received funding from the Veterans Affairs for research projects examining a low carbohydrate diet in patients with diabetes.

Provenance and peer review: Commissioned, externally peer reviewed

This article is one of a series commissioned by The BMJ. Open access fees for the series were funded by Swiss Re, which had no input in to the commissioning or peer review of the articles. The $B M J$ thanks the series advisers, Nita Forouhi and Dariush Mozaffarian, for valuable advice and guiding selection of topics in the series.

International Diabetes Federation . IDF diabetes atlas. 8th ed. IDF, 2017. www. diabetesatlas.org

2 Forouzanfar MH, Alexander L, Anderson HR, etal. GBD 2013 Risk Factors Collaborators. Global, regional, and national comparative risk assessment of 79 behavioural, environmental and occupational, and metabolic risks or clusters of risks in 188 countries, 1990-2013: a systematic analysis for the Global Burden of Disease Study 2013. Lancet 2015;386:2287-323. 10.1016/S0140-6736(15)00128-2 26364544

3 Pastors JG, Warshaw H, Daly A, Franz M, Kulkarni K. The evidence for the effectiveness of medical nutrition therapy in diabetes management. Diabetes Care 2002;25:608-13. 10.2337/diacare.25.3.608 11874956

4 Lianov L, Johnson M. Physician competencies for prescribing lifestyle medicine. JAMA 2010;304:202-3. 10.1001/jama.2010.903 20628134

5 Kahan S, Manson JE. Nutrition counseling in clinical practice: how clinicians can do better. JAMA 2017;318:1101-2. 10.1001/jama.2017.10434 28880975

6 Mohan V, Shah SN, Joshi SR, etal. DiabCare India 2011 Study Group. Current status of management, control, complications and psychosocial aspects of patients with diabetes in India: Results from the DiabCare India 2011 Study. Indian J Endocrinol Metab 2014:18:370-8. 10.4103/2230-8210.129715 24944934

7 Hippisley-Cox J, Pringle M. Prevalence, care, and outcomes for patients with diet-controlled diabetes in general practice: cross sectional survey. Lancet 2004;364:423-8. 10.1016/S0140-6736(04)16765-2 15288740

8 England $\mathrm{CY}$, Andrews RC, Jago R, Thompson JL. A systematic review of brief dietary questionnaires suitable for clinical use in the prevention and management of obesity, cardiovascular disease and type 2 diabetes. Eur J Clin Nutr 2015;69:977-1003. 10.1038/ejcn.2015.6 25711954

9 Miller V, Yusuf S, Chow CK, etal . Availability, affordability, and consumption of fruits and vegetables in 18 countries across income levels: findings from the Prospective Urban Rural Epidemiology (PURE) study. Lancet Glob Health 2016;4:e695-703. 10.1016/S2214-109X(16)30186-3 27567348

10 Mozaffarian D. Dietary and policy priorities for cardiovascular disease, diabetes, and obesity: a comprehensive review. Circulation 2016;133:187-225. 10.1161/CIRCULATIONAHA.115.018585 26746178

11 Ley SH, Hamdy O, Mohan V, Hu FB. Prevention and management of type 2 diabetes: dietary components and nutritional strategies. Lancet 2014;383:1999-2007. 10.1016/S0140-6736(14)60613-9 24910231

12 Evert AB, Boucher JL, Cypress M, etal. American Diabetes Association. Nutrition therapy recommendations for the management of adults with diabetes. Diabetes Care 2013;36:3821-42. 10.2337/dc13-2042 24107659

13 Dworatzek PD, Arcudi K, Gougeon R, Husein N, Sievenpiper JL, Williams SLCanadian Diabetes Association Clinical Practice Guidelines Expert Committee. Nutrition therapy. Can J Diabetes 2013:37(Suppl 1):S45-55. 10.1016/j.jcjd.2013.01.019 24070963

14 Ajala O, English P, Pinkney J. Systematic review and meta-analysis of different dietary approaches to the management of type 2 diabetes. Am J Clin Nutr 2013;97:505-16. 10.3945/ajcn.112.042457 23364002

15 Wheeler ML, Dunbar SA, Jaacks LM, etal . Macronutrients, food groups, and eating patterns in the management of diabetes: a systematic review of the literature, 2010. Diabetes Care 2012;35:434-45. 10.2337/dc11-2216 22275443

16 Dyson PA, Kelly T, Deakin T, etal. Diabetes UK Nutrition Working Group. Diabetes UK evidence-based nutrition guidelines for the prevention and management of diabetes. Diabet Med 2011;28:1282-8. 10.1111/j.1464-5491.2011.03371.x 21699560

17 Franz MJ, MacLeod J, Evert A, etal . Academy of Nutrition and Dietetics Nutrition Practice Guideline for Type 1 and Type 2 Diabetes in Adults: Systematic Review of Evidence for 
Medical Nutrition Therapy Effectiveness and Recommendations for Integration into the Nutrition Care Process. J Acad Nutr Diet 2017:117:1659-79.

10.1016/j.jand.2017.03.022 28533169

18 Schwingshackl L, Hoffmann G, Lampousi AM, etal . Food groups and risk of type 2 diabetes mellitus: a systematic review and meta-analysis of prospective studies. Eur $J$ Epidemiol 2017;32:363-75. 10.1007/s10654-017-0246-y 28397016

19 Mozaffarian D, Forouhi NG. Dietary guidelines and health-is nutrition science up to the task?BMJ 2018:360:k822. 10.1136/bmj.k822 29549076

20 Mann JI, De Leeuw I, Hermansen K, etal. Diabetes and Nutrition Study Group (DNSG) of the European Association. Evidence-based nutritional approaches to the treatment and prevention of diabetes mellitus. Nutr Metab Cardiovasc Dis 2004;14:373-94. 10.1016/S0939-4753(04)80028-0 15853122

21 Misra A, Sharma R, Gulati S, etal. National Dietary Guidelines Consensus Group. Consensus dietary guidelines for healthy living and prevention of obesity, the metabolic syndrome, diabetes, and related disorders in Asian Indians. Diabetes Technol Ther 2011;13:683-94. 10.1089/dia.2010.0198 21488798

22 Tay J, Luscombe-Marsh ND, Thompson $\mathrm{CH}$, etal . Comparison of low- and high-carbohydrate diets for type 2 diabetes management: a randomized trial. Am J Clin Nutr 2015;102:780-90. 10.3945/ajcn.115.112581 26224300

23 Tobias DK, Chen M, Manson JE, Ludwig DS, Willett W, Hu FB. Effect of low-fat diet interventions versus other diet interventions on long-term weight change in adults: a systematic review and meta-analysis. Lancet Diabetes Endocrinol 2015;3:968-79. 10.1016/S2213-8587(15)00367-8 26527511

24 Johnston BC, Kanters S, Bandayrel K, etal . Comparison of weight loss among named diet programs in overweight and obese adults: a meta-analysis. JAMA 2014;312:923-33. 10.1001/jama.2014.10397 25182101

25 Gardner CD, Kim S, Bersamin A, etal . Micronutrient quality of weight-loss diets that focus on macronutrients: results from the A TO Z study. Am J Clin Nutr 2010;92:304-12. 10.3945/ajcn.2010.29468 20573800

26 Gardner CD, Trepanowski JF, Del Gobbo LC, etal . Effect of low-fat vs low-carbohydrate diet on 12-month weight loss in overweight adults and the association with genotype pattern or insulin secretion: The DIETFITS Randomized Clinical Trial. JAMA 2018:319:667-79. 10.1001/jama.2018.0245 29466592

27 Wallin A, Di Giuseppe D, Orsini N, Patel PS, Forouhi NG, Wolk A. Fish consumption, dietary long-chain $\mathrm{n}-3$ fatty acids, and risk of type 2 diabetes: systematic review and meta-analysis of prospective studies. Diabetes Care 2012;35:918-29. $10.2337 / \mathrm{dc} 11-163122442397$

28 Aung T, Haynes R, Barton J, etal. ASCEND Study Collaborative Group. Cost-effective recruitment methods for a large randomised trial in people with diabetes: A Study of Cardiovascular Events in Diabetes (ASCEND). Trials 2016;17:286 10.1186/s13063-016-1354-9 27296091

29 Schwingshackl L, Lampousi AM, Portillo MP, Romaguera D, Hoffmann G, Boeing H. Olive oil in the prevention and management of type 2 diabetes mellitus: a systematic review and meta-analysis of cohort studies and intervention trials. Nutr Diabetes 2017;7:e262 10.1038/nutd.2017.12 28394365

30 Estruch R, Ros E, Salas-Salvadó J, etal. PREDIMED Study Investigators. Primary prevention of cardiovascular disease with a Mediterranean diet. N Engl J Med 2013;368:1279-90. 10.1056/NEJMoa1200303 23432189

31 Forouhi NG, Krauss RM, Taubes G, Willet W. Dietary fat and cardiometabolic health: evidence, controversies, and consensus for guidance. BMJ 2018;361:k2139.

32 Bero L. Developing reliable dietary guidelines. BMJ 2017;359:j4845 10.1136/bmj.j4845 29101096

33 Buse JB, Caprio S, Cefalu WT, etal . How do we define cure of diabetes?Diabetes Care 2009;32:2133-5. 10.2337/dc09-9036 19875608

34 McCombie L, Leslie W, Taylor R, Kennon B, Sattar N, Lean MEJ. Beating type 2 diabetes into remission. BMJ 2017;358:j4030. 10.1136/bmj.j4030 28903916

35 Turner RC, Cull CA, Frighi V, Holman RRUK Prospective Diabetes Study (UKPDS) Group. Glycemic control with diet, sulfonylurea, metformin, or insulin in patients with type 2 diabetes mellitus: progressive requirement for multiple therapies (UKPDS 49). JAMA 1999;281:2005-12. 10.1001/jama.281.21.2005 10359389

36 Guidone C, Manco M, Valera-Mora E, etal . Mechanisms of recovery from type 2 diabetes after malabsorptive bariatric surgery. Diabetes 2006;55:2025-31. 10.2337/db06-0068 16804072

37 Lim EL, Hollingsworth KG, Aribisala BS, Chen MJ, Mathers JC, Taylor R. Reversal of type 2 diabetes: normalisation of beta cell function in association with decreased pancreas and liver triacylglycerol. Diabetologia 2011;54:2506-14. 10.1007/s00125-011-2204-7 21656330

38 White MG, Shaw JA, Taylor R. Type 2 Diabetes: The Pathologic Basis of Reversible B-Cell Dysfunction. Diabetes Care 2016;39:2080-8. 10.2337/dc16-0619 2792689

39 Steven S, Hollingsworth KG, Al-Mrabeh A, etal . Very low-calorie diet and 6 months of weight stability in type 2 diabetes: pathophysiological changes in responders and nonresponders. Diabetes Care 2016;39:808-15. 10.2337/dc15-1942 27002059

40 Lean ME, Leslie WS, Barnes AC, etal. Primary care-led weight management for remission of type 2 diabetes (DiRECT): an open-label, cluster-randomised trial. Lancet 2018;391:541-51. 10.1016/S0140-6736(17)33102-1 29221645

41 Gregg EW, Chen H, Wagenknecht LE, etal. Look AHEAD Research Group. Association of an intensive lifestyle intervention with remission of type 2 diabetes. JAMA 2012;308:2489-96. 10.1001/jama.2012.67929 23288372

42 Peters C, Steven S, Taylor R. Case 73: Reversal of type 2 diabetes by weight loss despite presence of macro- and microvascular complications. In: Draznin B, ed. diabetes case studies real problems, practical solutions. American Diabetes Association, 2015:271-4.

43 Whittington J. Fixing dad. Short Books, 2016.

44 Pearce IA, Ilango B, Sells RA, Wong D. Stabilisation of diabetic retinopathy following simultaneous pancreas and kidney transplant. Br J Ophthalmol 2000;84:736-40. 10.1136/bjo.84.7.73610.1136/bjo.84.7.736 10873985

45 Arun CS, Pandit R, Taylor R. Long-term progression of retinopathy after initiation of insulin therapy in Type 2 diabetes: an observational study. Diabetologia 2004;47:1380-4. 10.1007/s00125-004-1473-9 15309288

46 The Kroc Collaborative Study Group. Diabetic retinopathy after two years of intensified insulin treatment. Follow-up of the Kroc Collaborative Study. JAMA 1988;260:37-41. 10.1001/jama.1988.03410010045032 2898025

47 Osler W, McCrae T. The principles and practice of medicine. D. Appleton and Company,
48 Westman EC, Yancy WSJr, Humphreys M. Dietary treatment of diabetes mellitus in the pre-insulin era (1914-1922). Perspect Biol Med 2006:49:77-83.

10.1353/pbm.2006.0017 16489278

49 Bisschop PH, De Sain-Van Der Velden MG, Stellaard F, etal . Dietary carbohydrate deprivation increases 24-hour nitrogen excretion without affecting postabsorptive hepatic or whole body protein metabolism in healthy men. J Clin Endocrinol Metab 2003;88:3801-5. 10.1210/jc.2002-021087 12915672

50 Boden G, Sargrad K, Homko C, Mozzoli M, Stein TP. Effect of a low-carbohydrate diet on appetite, blood glucose levels, and insulin resistance in obese patients with type 2 diabetes. Ann Intern Med 2005;142:403-11. 10.7326/0003-4819-142-6-200503150-00006 15767618

51 van Wyk HJ, Davis RE, Davies JS. A critical review of low-carbohydrate diets in people with Type 2 diabetes. Diabet Med 2016;33:148-57. 10.1111/dme.12964 26413954

52 Dyson P. Low Carbohydrate Diets and Type 2 Diabetes: What is the Latest Evidence?Diabetes Ther 2015;6:411-24. 10.1007/s13300-015-0136-9 26446553

53 Snorgaard O, Poulsen GM, Andersen HK, Astrup A. Systematic review and meta-analysis of dietary carbohydrate restriction in patients with type 2 diabetes. BMJ Open Diabetes Res Care 2017;5:e00354. 10.1136/bmjdrc-2016-000354 28316796

54 Kirk JK, Graves DE, Craven TE, Lipkin EW, Austin M, Margolis KL. Restricted-carbohydrate diets in patients with type 2 diabetes: a meta-analysis. J Am Diet Assoc 2008;108:91-100 . 10.1016/j.jada.2007.10.003 18155993

55 Meng Y, Bai H, Wang S, Li Z, Wang Q, Chen L. Efficacy of low carbohydrate diet for type 2 diabetes mellitus management: A systematic review and meta-analysis of randomized controlled trials. Diabetes Res Clin Pract 2017;131:124-31. 10.1016/j.diabres.2017.07.006 28750216

56 Schwingshackl L, Chaimani A, Hoffmann G, Schwedhelm C, Boeing H. A network meta-analysis on the comparative efficacy of different dietary approaches on glycaemic control in patients with type 2 diabetes mellitus. Eur J Epidemiol 2018;33:157-70. 10.1007/s10654-017-0352-x 29302846

57 Noakes TD, Windt J. Evidence that supports the prescription of low-carbohydrate high-fat diets: a narrative review. Br J Sports Med 2017;51:133-9. 10.1136/bjsports-2016-096491 28053201

58 Feinman RD, Pogozelski WK, Astrup A, etal . Dietary carbohydrate restriction as the first approach in diabetes management: critical review and evidence base. Nutrition 2015;31:1-13. 10.1016/..nut.2014.06.011 25287761

59 Hallberg SJ, McKenzie AL, Williams PT, etal . Effectiveness and safety of a novel care model for the management of type 2 diabetes at 1 year: an open-label, non-randomized, controlled study. Diabetes Ther 2018;9:583-612. 29417495

60 Hu T, Mills KT, Yao L, etal. Effects of low-carbohydrate diets versus low-fat diets on metabolic risk factors: a meta-analysis of randomized controlled clinical trials. Am J Epidemiol 2012;176(Suppl 7):S44-54. 10.1093/aje/kws264 23035144

61 Bueno NB, de Melo IS, de Oliveira SL, da Rocha Ataide T. Very-low-carbohydrate ketogenic diet v. low-fat diet for long-term weight loss: a meta-analysis of randomised controlled trials. Br J Nutr 2013;110:1178-87. 10.1017/S0007114513000548 23651522

62 Mansoor N Vinknes KJ, Veierød MB, Retterstøl K. Effects of low-carbohydrate diets v. low-fat diets on body weight and cardiovascular risk factors: a meta-analysis of randomised controlled trials. Br J Nutr 2016;115:466-79. 10.1017/S0007114515004699 26768850

63 Santos FL, Esteves SS, da Costa Pereira A, Yancy WSJr, Nunes JP. Systematic review and meta-analysis of clinical trials of the effects of low carbohydrate diets on cardiovascular risk factors. Obes Rev 2012;13:1048-66. 10.1111/j.1467-789X.2012.01021.x 22905670

64 Volek JS, Sharman MJ, Forsythe CE. Modification of lipoproteins by very low-carbohydrate diets. J Nutr 2005;135:1339-42. 10.1093/jn/135.6.1339 15930434

65 Friedman AN, Ogden LG, Foster GD, etal . Comparative effects of low-carbohydrate high-protein versus low-fat diets on the kidney. Clin J Am Soc Nephrol 2012;7:1103-11. 10.2215/CJN.11741111 22653255

66 Jesudason DR, Pedersen E, Clifton PM. Weight-loss diets in people with type 2 diabetes and renal disease: a randomized controlled trial of the effect of different dietary protein amounts. Am J Clin Nutr 2013:98:494-501. 10.3945/ajcn.113.060889 23719550

67 Brinkworth GD, Buckley JD, Noakes M, Clifton PM. Renal function following long-term weight loss in individuals with abdominal obesity on a very-low-carbohydrate diet vs high-carbohydrate diet. J Am Diet Assoc 2010;110:633-8. 10.1016/j.jada.2009.12.016 20338292

68 Reddy ST, Wang CY, Sakhaee K, Brinkley L, Pak CY. Effect of low-carbohydrate high-protein diets on acid-base balance, stone-forming propensity, and calcium metabolism. Am J Kidney Dis 2002;40:265-74. 10.1053/ajkd.2002.34504 12148098

69 Brinkworth GD, Wycherley TP, Noakes M, Buckley JD, Clifton PM. Long-term effects of a very-low-carbohydrate weight-loss diet and an isocaloric low-fat diet on bone health in obese adults. Nutrition 2016;32:1033-6. 10.1016/..nut.2016.03.003 27157472

70 India Go. Nutritional intake in India, 2011-12, NSS 68th Round. Ministry of Statistics and Programme Implementation, 2014.

71 Misra A, Singhal N, Sivakumar B, Bhagat N, Jaiswal A, Khurana L. Nutrition transition in India: secular trends in dietary intake and their relationship to diet-related non-communicable diseases. J Diabetes 2011;3:278-92. 10.1111/.1.1753-0407.2011.00139.x 21649865

72 Zhai F, Wang H, Du S, etal . Prospective study on nutrition transition in China. Nutr Rev 2009;67(Suppl 1):S56-61. 10.1111/j.1753-4887.2009.00160.x 19453679

73 Stern D, Piernas C, Barquera S, Rivera JA, Popkin BM. Caloric beverages were major sources of energy among children and adults in Mexico, 1999-2012. J Nutr 2014;144:949-56. 10.3945/jn.114.190652 24744311

74 Popkin BM. Synthesis and implications: China's nutrition transition in the context of changes across other low- and middle-income countries. Obes Rev 2014;15(Suppl 1):60-7. 10.1111/obr.12120 24341759

75 Bowen L, Ebrahim S, De Stavola B, etal . Dietary intake and rural-urban migration in India: a cross-sectional study. PLoS One 2011;6:e14822. 10.1371/journal.pone.0014822 21731604

76 Holmboe-Ottesen G, Wandel M. Changes in dietary habits after migration and consequences for health: a focus on South Asians in Europe. Food Nutr Res 2012;56:56. 10.3402/fnr.v56i0.1889123139649

77 Mente A, Dehghan M, Rangarajan S, etal. Prospective Urban Rural Epidemiology (PURE) study investigators. Association of dietary nutrients with blood lipids and blood pressure in 18 countries: a cross-sectional analysis from the PURE study. Lancet Diabetes Endocrinol 2017;5:774-87. 10.1016/S2213-8587(17)30283-8 28864143

78 Gulati S, Misra A. Abdominal obesity and type 2 diabetes in Asian Indians: dietary strategies including edible oils, cooking practices and sugar intake. Eur J Clin Nutr 2017;71:850-7. 10.1038/ejcn.2017.92 28612831 
79 Bhardwaj S, Passi SJ, Misra A, etal . Effect of heating/reheating of fats/oils, as used by Asian Indians, on trans fatty acid formation. Food Chem 2016;212:663-70. 10.1016/j.foodchem.2016.06.021 27374582

80 Downs SM, Singh A, Gupta V, Lock K, Ghosh-Jerath S. The need for multisectoral food chain approaches to reduce trans fat consumption in India. BMC Public Health 2015;15:693. 10.1186/s12889-015-1988-7 26197873

81 Tillin T, Sattar N, Godsland IF, Hughes AD, Chaturvedi N, Forouhi NG. Ethnicity-specific obesity cut-points in the development of type 2 diabetes - a prospective study including three ethnic groups in the United Kingdom. Diabet Med 2015;32:226-34 $10.1111 /$ dme.12576 25186015

82 World Health Organization. Sugars intake for adults and children. Guideline. WHO, 2015 http://www.who.int/nutrition/publications/guidelines/sugars_intake/en/
83 Scientific Advisory Committee on Nutrition. SACN Carbohydrates and Health Report. Public Health England. London, 2015. https://www.gov.uk/government/publications/sacncarbohydrates-and-health-report

Published by the BMJ Publishing Group Limited. For permission to use (where not already granted under a licence) please go to http://group.bmj.com/group/rights-licensing/ permissionsThis is an Open Access article distributed in accordance with the Creative Commons Attribution Non Commercial (CC BY-NC 4.0) license, which permits others to distribute, remix, adapt, build upon this work non-commercially, and license their derivative works on different terms, provided the original work is properly cited and the use is non-commercial. See: http://creativecommons.org/licenses/by-nc/4.0/. 


\section{Figure}

\section{In all populations}

Manage weight

\section{Specific considerations in management} of type 2 diabetes

Foods/overall diet quantity matters

quality is relevant

Focus on foods, not isolated nutrients

Consider overall eating patterns

General population

Coordinate dietary intake with type and dose of medication

In specific approaches such as low carbohydrate nutrition or very low calorie diets, monitor glycaemic control

Non-dietary factors are also important (eg, physical activity, smoking, other lifestyle factors)
Population at risk of type 2 diabetes

People with type 2 diabetes

Fig 1 Dietary advice for different populations for the prevention and management of type 2 diabetes 